\title{
Synthetic and pharmacological studies on some 1-isonicotinoyl-3- methyl-4-(4-substituted phenyl)-3a,4-dihydro pyrazolo [3,4-c]pyrazoles and their ethoxyphthalimide derivatives
}

\author{
Swati Ojha, Archita Bapna, and Ganpat L.Talesara* \\ Synthetic Organic Chemistry Laboratory, Department of Chemistry, M. L. Sukhadia University, \\ Udaipur (Raj.)- 313 001, India \\ E-mail: gtalesara@yahoo.com
}

\begin{abstract}
Isoniazid reacted with ethylacetoacetate in absolute ethanol to give 2-isonicotinoyl-5-methyl-2,4dihydro-3H-pyrazol-3-one (1), which upon condensation with various aldehydes (2a-d) afforded the related arylidene derivatives (3a-d). 1-Isonicotinoyl-3-methyl-4-(4-substitutedphenyl)-3a,4dihydropyrazolo[3,4-c]pyrazoles (4a-d) were obtained as the result of cyclocondensation reaction between 3a-d and hydrazine hydrate. Subsequently compounds $4 \mathbf{4 a - d}$ were converted to corresponding 2- $N$-ethoxyphthalimido-6-isonicotinoyl-4-methyl-3-(4-substituted phenyl)-3,3adihydro pyrazolo[3,4-c]pyrazoles 6a-d by the treatment with phthalimidoxyethyl bromide (5). Structures of synthesized compounds were elucidated by means of IR, ${ }^{1} \mathrm{H}$ NMR and mass spectral data. Final compounds were screened for four biological activities namely "antibacterial, antifungal, antiviral and anticancer".
\end{abstract}

Keywords: Isoniazid, pyrazolo[3,4-c]pyrazoles, phthalimidoxyethyl bromide, antifungal, spectral data

\section{Introduction}

Isoniazid is reported as a well known and well acknowledged drug. ${ }^{1-3}$ Isoniazid is one of the primary drugs used in combination with ethanbutol, rifampin, streptomycin and pyrazinamide to treat tuberculosis. ${ }^{4}$ Despite the large number of compounds containing the isoniazid moiety which have already been synthesized and tested, there is still a need for new compounds of this kind, ${ }^{5}$ due to the increasing resistance of bacterial strains of certain type of antibiotics. ${ }^{6}$ The efficiency of pyrazole as chemotherapeutic agent is well established and their chemistry has been extensively studied. Pyrazole and its synthetic analogues have been found to exhibit industrial, agricultural and biological applications. ${ }^{7-11}$ Pyrazoles are an interesting group of compounds many of which possess broad spectrum pharmacological properties, such as analgesic, 
antipyretic, antidepressant and antirheumatic ${ }^{12,13}$ and are also well known for their pronounced anti-inflammatory activity ${ }^{14}$ as well they are used as potent antidiabetic agents ${ }^{15}$. Moreover, pyrazoles have played a crucial part in the development of heterocyclic chemistry and used extensively as useful synthon in organic synthesis. ${ }^{16-19}$

Alkoxyphthalimides are a class of compounds well known for a long time and still continue the object of considerable interest, mainly due to their applications in different fields particularly as pharmaceuticals. Mainly these are used as potent anticancer ${ }^{20}$ and anticonvulsant ${ }^{21-22}$ agents. In addition, presence of pyridine ring is conducive to improvements of the biological activity. These valid observations regarding the pharmacological importance of the above mentioned moieties and in connection with our on going programme of synthesizing alkoxyphthalimide derivatives $^{23-25}$ of certain heterocyclic systems led us to undertake the synthesis of some new combinational molecules, incorporating above moieties in them with the hope of augmentation in biological activities

\section{Results and Discussion}

To prepare 2-isonicotinoyl-5-methyl-2,4-dihydro-3H-pyrazol-3-one (1), isoniazid (isonicotinic acid hydrazide) was treated with ethylacetoacetate in absolute alcohol. Alternatively, (1) was prepared by the reaction of isoniazid and ethylacetoacetate in presence of sodium ethoxide solution in ethyl alcohol. Presence of base reduces the reaction time but yields are not satisfactory. So the base free method is preferred, although it takes a longer time. Formation of (1) is confirmed by the disappearance of bands near 3300-3400 $\mathrm{cm}^{-1}$ for $\mathrm{NH}_{2}$ functionality and presence of a band near $2973 \mathrm{~cm}^{-1}$ for methylene and methyl group. Analysis of its ${ }^{1} \mathrm{H}$ NMR spectrum revealed the signals of aromatic proton near $\delta 7.99-8.79$, which confirmed the presence of pyridine nucleus in it. Transformation of 2-isonicotinoyl-5-methyl-2,4-dihydro-3H-pyrazol-3one (1) to its corresponding arylidene derivatives (3a-d) was achieved by its treatment with various 4-substituted benzaldehydes (2a-d). Structure of these compounds was established spectroscopically, i.e. presence of a singlet at $\delta 6.5$ (3a) for methine proton.

Compounds (3a-d) when were subjected to reaction with hydrazine hydrate, a cyclocondensation reaction afforded 4-(4-chlorophenyl/4-methoxyphenyl/4- $N, N$ dimethylaminophenyl/phenyl)-1-isonicotinoyl-3-methyl-3a,4-dihydropyrazolo[3,4-c]- pyrazole (4a-d). The structures of these compounds were determined from their analytical and spectral data. The IR absorptions due to the N-H function appeared at $3430 \mathrm{~cm}^{-1}$. The absorption bands associated with other functionalities present all appeared in the expected regions. The ${ }^{1} \mathrm{H} N M R$ spectra of the compound (4a) exhibited a sharp singlet at $\delta 6.23$ corresponding to the NH proton of the pyrazole ring. The N-H proton of (4a-d) was replaced from ethoxyphthalimide moiety by the reaction with phthalimidoxyethyl bromide (5).

The resultant product was identified as 2- $N$-ethoxyphthalimido-6-isonicotinoyl-4-methyl-3(4-substituted phenyl)-3,3a-dihydro pyrazolo[3,4-c]pyrazoles (6a-d). The IR spectra of (6a) 
display strong absorption band for CO-N-CO group at around 1789-1728 $\mathrm{cm}^{-1}$, while $\mathrm{N}-\mathrm{O}$ and C-O bond give relatively weak absorption bands at 1493 and $1079 \mathrm{~cm}^{-1}$ respectively. Disappearance of $\mathrm{NH}$ stretching band around $3460 \mathrm{~cm}^{-1}$ also confirmed the replacement of hydrogen of pyrazole NH by ethoxyphthalimide, which was present in its precursor. Additional proof for the proposed structure of (6a-d) was provided by close observation of ${ }^{1} \mathrm{H} N M R$ spectra, which showed disappearance of $\mathrm{NH}$ signal at $\delta 6.2$ and presence of new signals for $\mathrm{NCH}_{2}$ and $\mathrm{OCH}_{2}$ protons resonating at $\delta 3.64$ and 4.50 respectively.

\section{REACTION SCHEME}

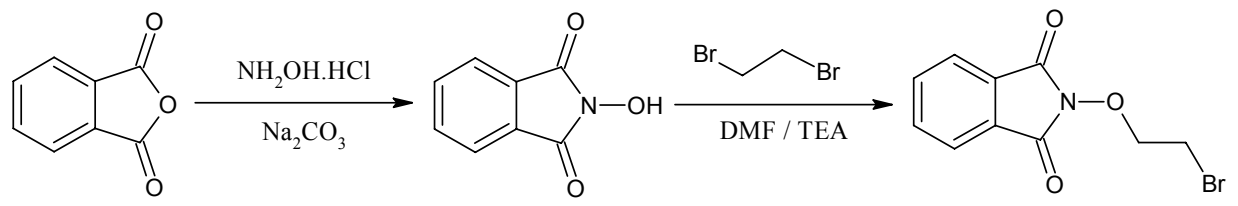

(5)
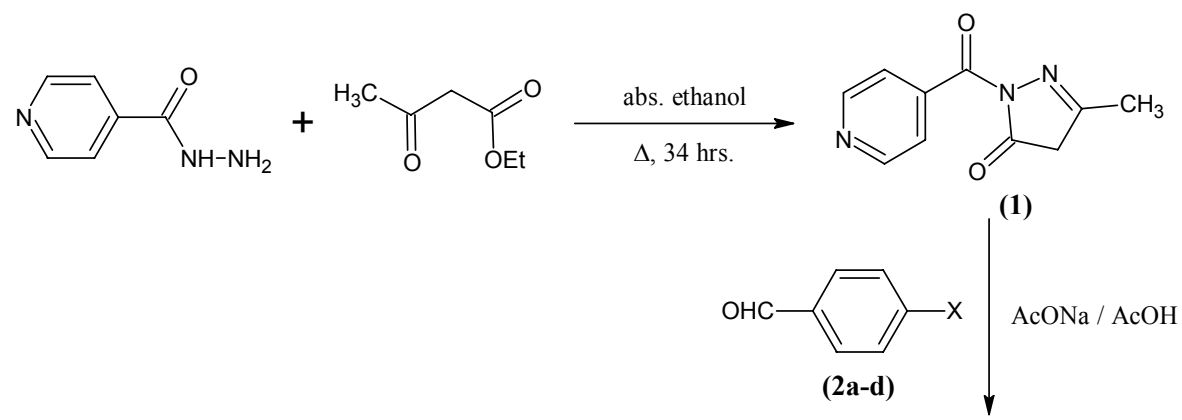<smiles>CC1=NN(C(=O)c2ccncc2)C2=NNC(c3ccc(C)cc3)C12</smiles>

(4a-d)

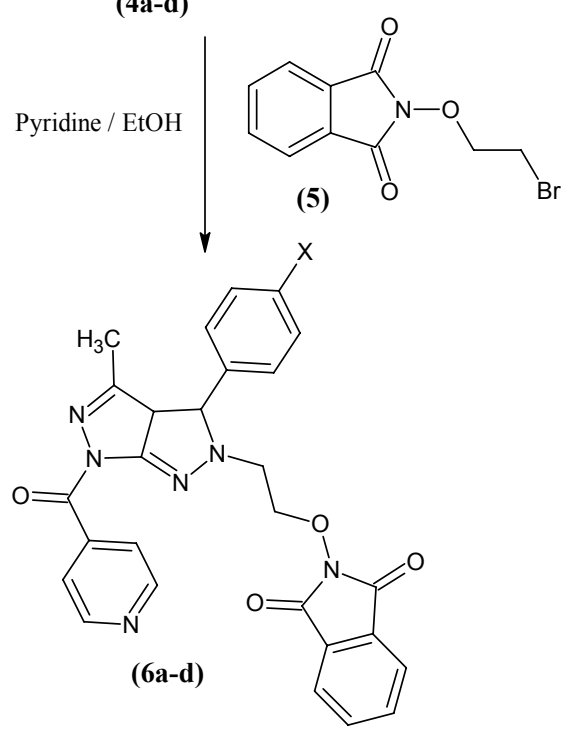

$\mathrm{NH}_{2} \mathrm{NH}_{2} \cdot \mathrm{H}_{2} \mathrm{O}$<smiles>[X]c1ccc(/C=C2\C(=O)N(C(=O)c3ccncc3)N=C2C)cc1</smiles>

$\begin{array}{ccccc} & \text { a } & \text { b } & \text { c } & \text { d } \\ \mathbf{X} & \mathrm{Cl} & \mathrm{OCH}_{3} & \mathrm{~N}\left(\mathrm{CH}_{3}\right)_{2} & \mathrm{H}\end{array}$ 


\section{Antimicrobial screening}

Antimicrobial activity, i.e. antibacterial and antifungal was screened by the Well or Cup method $^{27}$ in nutrient agar and dextrose agar medium. The agar medium was sterilized by autoclaving at $15 \mathrm{psi}$ and $121{ }^{\circ} \mathrm{C}$ for twenty minutes. The medium was poured in Petri dishes and left to solidify. These Petri dishes were inoculated with $0.2 \mathrm{~mL}$ suspension of organism by spread plate method ${ }^{28}$. Three or four wells of $11 \mathrm{~mm}$ diameter were made in the medium with the help of a sterile borer and filled with 500 ppm solution of testing compound in DMF. Similarly other wells were made for standard drugs and filled with standard concentration. These Petri plates were incubated at $37{ }^{0} \mathrm{C}$ in an incubator. The Petri dishes were examined for zone of inhibition after 24-48 hrs. For the present investigation; one gram positive- $B$. subtilis and three gram negative strains $P$. mirabilis, E. coli and $K$. pneumoniae were used. Two standard drugs were used for comparative study viz. ciprofloxacin and roxithromycin. Candida albicans (MTCC227) and Aspergillus fumigatus (MTCC2550) were used as the testing fungal strains. Amphotericin B and flucanazole were used as standard drugs. Zone of inhibition is measured in $\mathrm{mm}$. Activity index of all the synthesized compounds was also calculated against all the standard drugs.

Compounds (4a-d) and their ethoxyphthalimide derivatives (6a-d) were assayed for antimicrobial activity. Screening results of the compounds (4a-d) established that the (4b) showed comparable activity against all the tested micro-organisms as compared to the standard drugs used, while (4a) exhibited good activity and (4c) \& (4d) were either inactive or weakly active against all the four bacterial and two fungal strains. Overall activity profile of compounds (4a-d) was found to be moderate to poor. Ciprofloxacin was found to be stronger drug than roxithromycin.

Therefore in the present study, we attempted to increase antimicrobial activities by fusing the ethoxyphthalimide moiety with the pyrazolo[3,4-c]pyrazole ring system. Compound (6b) was found to be the strongest amongst all. Compounds have more comprehensive fungus-inhibiting properties than that of the bacterial. Even three to four folds antifungal activity was observed than standards. Compound (6a) and (6b) showed strong activity against P. mirabilis, B. subtilis, $C$. albicans and A. fumigatus while moderate to good activity against $K$. pneumoniae and $E$. coli. The effect of incorporation of ethoxyphthalimide moiety to the pyrazole ring system was much pronounced on their activity. As far as the relation between structure and activity are concerned the chloro and methoxy substituted compounds were found to show better activity than the others. 
Table 1. Antimicrobial activity of the synthesized compounds (4a-d) and (6a-d). Zone of inhibition (mm) (activity index $)^{\text {std. }}$

\begin{tabular}{|c|c|c|c|c|c|c|}
\hline \multirow[b]{2}{*}{$\begin{array}{c}\text { Compd. } \\
\text { No. }\end{array}$} & \multicolumn{4}{|c|}{ Antibacterial activity } & \multicolumn{2}{|c|}{ Antifungal activity } \\
\hline & $\begin{array}{c}P . \\
\text { mirabilis }\end{array}$ & $\begin{array}{c}K . \\
\text { pneumoniae }\end{array}$ & $\begin{array}{c}E . \\
\text { coli }\end{array}$ & $\begin{array}{c}\text { B. } \\
\text { subtilis }\end{array}$ & $\begin{array}{c}C . \\
\text { albicans }\end{array}$ & $\begin{array}{c}A . \\
\text { fumigatus }\end{array}$ \\
\hline $4 \mathbf{a}$ & $\begin{array}{c}8 \\
(0.57)^{C_{1}} \\
(0.61)^{C}{ }_{2}\end{array}$ & $\begin{array}{c}9 \\
(0.75)^{C_{1}} \\
(1.5)^{C}{ }_{2}\end{array}$ & $\begin{array}{c}5 \\
(0.41)^{C_{1}} \\
(0.50)^{C_{2}}\end{array}$ & $\begin{array}{c}10 \\
(0.90)^{C_{1}} \\
(1.66)^{C_{2}}\end{array}$ & $\begin{array}{c}13 \\
(1.08)^{C_{1}} \\
(2.16)^{C}{ }_{2}\end{array}$ & $\begin{array}{c}14 \\
(2.33)^{C_{1}} \\
(4.66)^{C_{2}}\end{array}$ \\
\hline $4 b$ & $\begin{array}{c}10 \\
(0.71)^{C_{1}} \\
(1.00)^{C_{2}}\end{array}$ & $\begin{array}{c}6 \\
(0.50)^{C_{1}} \\
(1.00)^{C_{2}}\end{array}$ & $\begin{array}{c}5 \\
(0.41)^{C_{1}} \\
(0.50)^{C_{2}}\end{array}$ & $\begin{array}{c}9 \\
(0.81)^{\mathrm{C}_{1}} \\
(1.5)^{\mathrm{C}}{ }_{2}\end{array}$ & $\begin{array}{c}15 \\
(1.25)^{\mathrm{C}_{1}} \\
(2.5)^{\mathrm{C}}{ }_{2}\end{array}$ & $\begin{array}{c}17 \\
(2.83){ }^{C_{1}} \\
(5.66){ }^{C}{ }_{2}\end{array}$ \\
\hline $4 c$ & $\begin{array}{c}8 \\
(0.57)^{C_{1}} \\
(0.80)^{C_{2}}\end{array}$ & $\begin{array}{c}8 \\
(0.66)^{C_{1}} \\
(1.33){ }^{C_{2}}\end{array}$ & $\begin{array}{c}4 \\
(0.33)^{C_{1}} \\
(0.40)^{C_{2}}\end{array}$ & $\begin{array}{c}7 \\
(0.63)^{C_{1}} \\
(1.16)^{C_{2}}\end{array}$ & $\begin{array}{c}12 \\
(1.00)^{C_{1}} \\
(2.00)^{C_{2}}{ }_{2}\end{array}$ & $\begin{array}{c}13 \\
(2.16)^{C_{1}} \\
(4.33)^{C_{2}}\end{array}$ \\
\hline $4 d$ & $\begin{array}{c}6 \\
(0.42)^{C_{1}} \\
(0.60)^{C_{2}}\end{array}$ & $\begin{array}{c}7 \\
(0.58)^{C_{1}} \\
(1.16)^{C_{2}}\end{array}$ & NA & $\begin{array}{c}4 \\
(0.36)^{C_{1}} \\
(0.66)^{C_{2}}\end{array}$ & $\begin{array}{c}10 \\
(0.83)^{C_{1}} \\
(1.66)^{C_{2}}\end{array}$ & $\begin{array}{c}9 \\
(1.5)^{C_{1}} \\
(3.00)^{C_{2}}\end{array}$ \\
\hline $\mathbf{6 a}$ & $\begin{array}{c}13 \\
(0.92)^{C_{1}} \\
(1.3)^{C_{2}}\end{array}$ & $\begin{array}{c}10 \\
(0.83)^{C_{1}} \\
(1.66)^{C}{ }_{2}\end{array}$ & $\begin{array}{c}7 \\
(0.58){ }^{C_{1}} \\
(0.70)^{C_{2}}\end{array}$ & $\begin{array}{c}14 \\
(1.27)^{C_{1}} \\
(2.33)^{C_{2}}\end{array}$ & $\begin{array}{c}21 \\
(1.75)^{C_{1}} \\
(3.5)^{C_{2}}\end{array}$ & $\begin{array}{c}19 \\
(3.16)^{C_{1}} \\
(6.33)^{C_{2}}\end{array}$ \\
\hline $6 \mathbf{b}$ & $\begin{array}{c}15 \\
(1.07)^{C_{1}} \\
(1.5)^{C_{2}}\end{array}$ & $\begin{array}{c}11 \\
(0.91)^{C_{1}} \\
(1.83)^{C_{2}}\end{array}$ & $\begin{array}{c}8 \\
(0.66)^{C_{1}} \\
(0.80)^{C_{2}}\end{array}$ & $\begin{array}{c}12 \\
(1.09)^{C_{1}} \\
(2.00)^{C_{2}}\end{array}$ & $\begin{array}{c}22 \\
(1.83)^{C_{1}} \\
(3.66)^{C_{2}}\end{array}$ & $\begin{array}{c}18 \\
(3.00)^{\mathrm{C}_{1}} \\
(6.00)^{\mathrm{C}_{2}}\end{array}$ \\
\hline $6 c$ & $\begin{array}{c}14 \\
(1.00)^{C_{1}} \\
(1.4)^{C_{2}}\end{array}$ & $\begin{array}{c}9 \\
(0.75)^{C_{1}} \\
(1.5)^{C_{2}}\end{array}$ & $\begin{array}{c}6 \\
(0.50)^{C_{1}} \\
(0.60)^{C_{2}}\end{array}$ & $\begin{array}{c}13 \\
(1.18)^{C_{1}} \\
(2.16)^{C_{2}}\end{array}$ & $\begin{array}{c}19 \\
(1.58)^{C_{1}} \\
(3.16)^{C_{2}}\end{array}$ & $\begin{array}{c}15 \\
(2.5)^{\mathrm{C}_{1}} \\
(5.00)^{\mathrm{C}}{ }_{2}\end{array}$ \\
\hline $6 d$ & $\begin{array}{c}11 \\
(0.78)^{C_{1}} \\
(1.1)^{C_{2}}\end{array}$ & $\begin{array}{c}7 \\
(0.58)^{C_{1}} \\
(1.16)^{C_{2}}\end{array}$ & $\begin{array}{c}6 \\
(0.50)^{C_{1}} \\
(0.60)^{C_{2}}\end{array}$ & $\begin{array}{c}12 \\
(1.09)^{C_{1}} \\
(2.00)^{C_{2}}\end{array}$ & $\begin{array}{c}14 \\
(1.16)^{C_{1}} \\
(2.33)^{C_{2}}\end{array}$ & $\begin{array}{c}12 \\
(2.00){ }^{C_{1}} \\
(4.00){ }^{C_{2}}\end{array}$ \\
\hline $\mathbf{C}_{1}$ & 14 & 12 & 12 & 11 & 12 & 6 \\
\hline $\mathbf{C}_{2}$ & 10 & 6 & 10 & 6 & 6 & 3 \\
\hline
\end{tabular}

(Activity index $)=$ Inhibition zone of compound/Inhibition zone of the standard drug.

For antibacterial activity: $\mathrm{C}_{1}=$ Ciprofloxacin, $\mathrm{C}_{2}=$ Roxithromycin

For antifungal activity: $\mathrm{C}_{1}=$ Amphotericin $\mathrm{B}, \mathrm{C}_{2}=$ flucanazole

NA $=$ Negligible Activity

\section{Anticancer screening ${ }^{29-30}$}

All the four Ethoxyphthalimide derivatives of Isonicotinoyl pyrazolo[3,4-c]pyrazoles (6a-d) have been assayed for the antiproliferative effects against murine leukemia cells (L1210/0) and 
human T-lymphocyte cells (Molt 4/C8, CEM/0 cells). Their $\mathrm{IC}_{50}$ values $(50 \%$ inhibitor concentration) were measured in $\mu \mathrm{g} / \mathrm{mL}$. None of the compounds exhibited antitumor cell activity at a reasonable low concentration (about $20 \mu \mathrm{g} / \mathrm{mL}$ ).

\section{Antiviral testing}

Four final compounds were screened for antiviral activity against influenza A (H1N1 and H3N2 subtypes) and influenza B viruses and cytotoxicity in MDCK (Madin Darby Canine Kidney) cells. Results have been expressed in the form of $\mathrm{EC}_{50}$ values (Effective concentration or concentration producing $50 \%$ inhibition of virus-induced cytopathic effects as determined by visual scoring of the CPE or by measuring the cell viability with the colorimetric formazan based MTS assay).

The reference compounds oseltavimir carboxylate and ribavirin were active against influenza virus; their $\mathrm{EC}_{50}$ values are clearly lower than their $\mathrm{MCC}$ value (concentrations causing minimal toxicity). For amantadin and rimantidin, the best activity was seen with the H3N2 strain. These compounds are known to be inactive against influenza $\mathrm{B}$. Also the $\mathrm{H} 1 \mathrm{~N} 1 \mathrm{~A} / \mathrm{PR} / 8 / 34$ strain that is used in tests is known to be less sensitive to amantadin and rimantadin. Among the range of four compounds tested, none was able to inhibit the cytopathic effects of influenza A or B at subtoxic concentration or at the highest concentration tested $(100 \mu \mathrm{g} / \mathrm{mL})$.

\section{Cytotoxicity and antiviral activity of synthesized compounds in HEL, HeLa and Vero cell cultures}

Antiviral assay in HEL, HeLa and Vero cells with the four compounds (6a-d) have been observed on Herpes simplex virus-1 \& 2, Vaccinia virus, Vesicular stomatitis virus, Coxsackie virus B4, Respiratory syncytical virus, Para-influenza-3 virus, Reovirus-1 and Puntatoro virus. No specific antiviral effects (i.e. minimal antivirally effective concentration) were noted for any of the compounds evaluated against any of the viruses.

\section{Experimental Section}

General Procedures. Melting points were taken in open capillary tubes and are therefore uncorrected. Purity of the compounds was checked on silica gel G TLC plates of $2 \mathrm{~mm}$ thickness using n-hexane and ethylacetate as solvent system. The visualization of spot was carried out in an iodine chamber. The IR spectra of the compounds were recorded in the $4000-450 \mathrm{~cm}^{-1}$ ranges using $\mathrm{KBr}$ discs on FTIR IR RX1 Perkin Elmer spectrophotometers and ${ }^{1} \mathrm{H}$ NMR were recorded on a Bruker DRX-300 MHz spectrometer $\left(\mathrm{CDCl}_{3}\right)$ using TMS as an internal standard. The ESIMS were recorded on a MICROMASS QUATTRO II triple quadrupole mass spectrometer having a JASCOPU-980 HPLC pump connected to it. 
Synthesis of phthalimidoxyethyl bromide (5). ${ }^{\mathbf{2 6}}$ A solution of N-hydroxyphthalimide $(0.1$ mol) in dimethyl formamide was prepared and 1,2-dibromoethane $(0.2 \mathrm{~mol})$ and triethylamine $(0.02 \mathrm{~mol})$ was added to it. This was allowed to stand at room temperature with occasional stirring, until the red colour of the mixture turned colourless (18 hrs.). The precipitate of triethylammonium bromide was filtered at suction. The filtrate was diluted with ice cold water $(800 \mathrm{~mL})$ and the solid precipitated was filtered off. The crude product was recrystallized by ethanol. M.P. $79{ }^{0} \mathrm{C}$, yield $52 \%$.

Synthesis of 2-isonicotinoyl-5-methyl-2,4-dihydro-3H-pyrazol-3-one (1). A mixture of isoniazid (isonicotinic acid hydrazide) $(0.01 \mathrm{~mol})$ and ethylacetoacetate was taken in absolute alcohol $(30 \mathrm{~mL})$ and refluxed for $34 \mathrm{hrs}$. Excess of solvent was distilled off and the resultant residue was poured on crushed ice to obtain the yellow long needle shaped crystals of 1. 1: yield 89\%, m.p. $>300{ }^{0} \mathrm{C}$; IR (KBr) cm ${ }^{-1}$ : 3110 (C-H str., Ar-H), 2973 (C-H str., $\left.\mathrm{CH}_{3}\right), 1683(\mathrm{C}=\mathrm{O}$ str.), 1553 (C=N str.); ${ }^{1} \mathrm{H}$ NMR $\left(\mathrm{CDCl}_{3}\right)$ 8: 7.99-8.79 (m, 4H, Ar-H), 4.25 (s, 2H, $\left.\mathrm{CH}_{2}\right), 2.01$ (s, $3 \mathrm{H}, \mathrm{CH}_{3}$ ); Anal. Calcd. for $\mathrm{C}_{10} \mathrm{H}_{9} \mathrm{~N}_{3} \mathrm{O}_{2}$ : C, 59.11; H, 4.46; N, 20.68. Found : C, 59.02; H, 4.37; N, $20.42 \%$.

Synthesis of 4-(4-chlorobenzylidene)-2-isonicotinoyl-5-methyl-2,4-dihydro-3H-pyrazol-3one (3a). Compound (1, $0.01 \mathrm{~mol})$ was dissolved in acetic acid. p-Chlorobenzaldehyde (2a-d, $0.01 \mathrm{~mol})$ and sodium acetate $(0.01 \mathrm{~mol})$ were also added to it. The resultant reaction mixture was now refluxed for 10 hrs., cooled, filtered, poured on crushed ice and kept for some time. After 23 hrs., orange coloured long crystals of 4-(4-chlorobezylidene)-2-isonicotinoyl-5-methyl-2,4dihydro-3H-pyrazol-3-one slowly appeared. It was filtered and dried for further reaction. 3a: yield 78\%, m.p. $260{ }^{0} \mathrm{C}$; IR (KBr) cm ${ }^{-1}: 3092$ (C-H str., Ar-H), 2980 (C-H str., $\left.\mathrm{CH}_{3}\right), 1680(\mathrm{C}=\mathrm{O}$ str.), 1585 (C=N str.), 746 (C-Cl str.); ${ }^{1} \mathrm{H} \mathrm{NMR}\left(\mathrm{CDCl}_{3}\right) \delta: 7.50-8.88$ (m, 8H, Ar-H), 6.5 (s, 1H, $=\mathrm{CH}-\mathrm{Ar}), 2.22\left(\mathrm{~s}, 3 \mathrm{H}, \mathrm{CH}_{3}\right)$; Anal. Calcd. for $\mathrm{C}_{17} \mathrm{H}_{12} \mathrm{~N}_{3} \mathrm{O}_{2} \mathrm{Cl}: \mathrm{C}, 62.68 ; \mathrm{H}, 3.31 ; \mathrm{N}, 12.90$. Found: C, 62.39; H, 3.58; N, $12.83 \%$.

Similarly, compounds (3b-d) were prepared with some change in reflux time and reaction work up. Their characteristic spectral and analytical data are given below:

2-Isonicotinoyl-4-(4-methoxybenzylidene)-5-methyl-2,4-dihydro-3H-pyrazol-3-one

(3b). Yield 80\%, m.p. $254{ }^{0} \mathrm{C}$; IR (KBr) cm ${ }^{-1}$ : 3080 (C-H str., Ar-H), $2982\left(\mathrm{C}-\mathrm{H}\right.$ str., $\left.\mathrm{CH}_{3}\right), 1672(\mathrm{C}=\mathrm{O}$ str.), 1590 (C=N str.), 1080 (C-O str.); ${ }^{1} \mathrm{H}$ NMR $\left(\mathrm{CDCl}_{3}\right) \delta: 7.03-8.70$ (m, 8H, Ar-H), 6.65 (s, $1 \mathrm{H},=\mathrm{CH}-\mathrm{Ar}), 3.83\left(\mathrm{~s}, 3 \mathrm{H}, \mathrm{OCH}_{3}\right), 2.22\left(\mathrm{~s}, 3 \mathrm{H}, \mathrm{CH}_{3}\right)$; Anal. Calcd. for $\mathrm{C}_{18} \mathrm{H}_{15} \mathrm{~N}_{3} \mathrm{O}_{3}: \mathrm{C}, 67.28 ; \mathrm{H}$, 4.71; N, 13.08. Found: C, 67.19; H, 4.60; N, 13.01\%.

4-(4-N,N-Dimethylaminobenzylidene)-2-isonicotinoyl-5-methyl-2,4-dihydro-3H-pyrazol-3one (3c). Yield 71\%, m.p. $271{ }^{0} \mathrm{C}$; IR (KBr) cm ${ }^{-1}$ : 3095 (C-H str., Ar-H), 2970 (C-H str., $\mathrm{CH}_{3}$ ), 1670 (C=O str.), 1600 (C=N str.); ${ }^{1} \mathrm{H}$ NMR $\left(\mathrm{CDCl}_{3}\right) \delta: 7.42-8.55$ (m, 8H, Ar-H), 6.68 (s, 1H, $=\mathrm{CH}-\mathrm{Ar}), 3.27\left(\mathrm{~s}, 6 \mathrm{H}, \mathrm{N}\left(\mathrm{CH}_{3}\right)_{2}\right), 2.20\left(\mathrm{~s}, 3 \mathrm{H}, \mathrm{CH}_{3}\right)$; Anal. Calcd. for $\mathrm{C}_{19} \mathrm{H}_{18} \mathrm{~N}_{4} \mathrm{O}_{2}$ : C, 68.25; $\mathrm{H}$, 5.43; N, 16.76. Found: C, 68.22; H, 5.28; N, 16.60\%.

4-Benzylidene-2-isonicotinoyl-5-methyl-2,4-dihydro-3H-pyrazol-3-one (3d). Yield 75\%, m.p. $240{ }^{0} \mathrm{C}$; IR (KBr) cm ${ }^{-1}$ : 3100 (C-H str., Ar-H), 2972 (C-H str., $\left.\mathrm{CH}_{3}\right), 1690(\mathrm{C}=\mathrm{O}$ str. $), 1602(\mathrm{C}=\mathrm{N}$ 
str.); ${ }^{1} \mathrm{H}$ NMR $\left(\mathrm{CDCl}_{3}\right) \delta: 7.29-8.80(\mathrm{~m}, 9 \mathrm{H}, \mathrm{Ar}-\mathrm{H}), 6.42(\mathrm{~s}, 1 \mathrm{H},=\mathrm{CH}-\mathrm{Ar}), 2.20\left(\mathrm{~s}, 3 \mathrm{H}, \mathrm{CH}_{3}\right)$; Anal. Calcd. for $\mathrm{C}_{17} \mathrm{H}_{13} \mathrm{~N}_{3} \mathrm{O}_{2}$ : C, 70.09; H, 4.50; N, 14.42. Found: C, 70.01; H, 4.39; N, 14.31\%. Synthesis of 4-(4-chlorophenyl)-1-isonicotinoyl-3-methyl-3a,4-dihydropyrazolo[3,4c)pyrazole (4a). Compound (3a, $0.01 \mathrm{~mol})$ and hydrazine hydrate $(0.02 \mathrm{~mol})$ were taken in absolute alcohol and a few drops of acetic acid were added to it as catalyst. It was then refluxed for $8 \mathrm{hrs}$, concentrated, cooled and poured on crushed ice. The product obtained was washed several times with water and then dried. 4a: Yield 65\%, m.p. $280{ }^{0} \mathrm{C}$; IR $(\mathrm{KBr}) \mathrm{cm}^{-1}: 3430(\mathrm{~N}-\mathrm{H}$ str.), 3048 (C-H str., Ar-H), 2996, 2853 (C-H str., $\mathrm{CH}_{3}$ ), 1624 (C=O str.), 1589 (C=N str.), 819 (C-Cl str.); ${ }^{1} \mathrm{H}$ NMR $\left(\mathrm{CDCl}_{3}\right) \delta: 7.41-8.60$ (m, 8H, Ar-H), 6.23 (s, 1H, NH), 4.30-4.31 (dd, 2H, $\mathrm{CH}-\mathrm{CH}$ ), 2.19 (s, 3H, $\mathrm{CH}_{3}$ ); Anal. Calcd. for $\mathrm{C}_{17} \mathrm{H}_{14} \mathrm{~N}_{5} \mathrm{OCl}$ : C, 60.09; H, 4.15; N, 20.61. Found: C, 60.12; H, 4.08; N, 20.52\%.

Likewise, compounds (4b-d) were prepared with some change in reaction conditions. Their characteristic spectral and analytical data are given below:

1-Isonicotinoyl-4-(4-methoxyphenyl)-3-methyl-3a,4-dihydropyrazolo[3,4-c]pyrazole (4b). Yield 68\%, m.p. $272{ }^{0} \mathrm{C}$; IR (KBr) cm ${ }^{-1}: 3418$ (N-H str.), 3052 (C-H str., Ar-H), 2980, 2840 (C$\mathrm{H}$ str., $\mathrm{CH}_{3}$ ), 1622 (C=O str.), 1580 (C=N str.), 1084 (C-O str.); ${ }^{1} \mathrm{H}$ NMR $\left(\mathrm{CDCl}_{3}\right) \delta: 7.1-8.61$ (m, 8H, Ar-H), 6.23 (s, 1H, NH), 4.32 (dd, 2H, CH-CH), 3.47 (s, 3H, OCH $), 2.10\left(\mathrm{~s}, 3 \mathrm{H}, \mathrm{CH}_{3}\right)$; Anal. Calcd. for $\mathrm{C}_{18} \mathrm{H}_{17} \mathrm{~N}_{5} \mathrm{O}_{2}$ : C, 64.47; H, 5.11; N, 20.88. Found: C, 64.36; H, 5.00; N, 20.81\%.

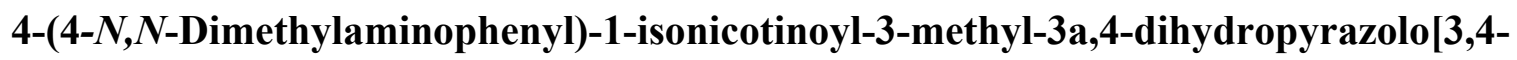
c]pyrazole (4c). Yield 60\%, m.p. $>300{ }^{0} \mathrm{C}$; IR (KBr) cm $\mathrm{cm}^{-1}: 3412$ (N-H str.), 3060 (C-H str., Ar$\mathrm{H}), 2960,2870$ (C-H str., $\left.\mathrm{CH}_{3}\right), 1620$ (C=O str.), 1582 (C=N str.); ${ }^{1} \mathrm{H}$ NMR $\left(\mathrm{CDCl}_{3}\right) \delta: 7.1-8.62$ (m, 8H, Ar-H), 6.20 (s, 1H, NH), $4.34(\mathrm{dd}, 2 \mathrm{H}, \mathrm{CH}-\mathrm{CH}), 2.82\left(\mathrm{~s}, 6 \mathrm{H}, \mathrm{N}\left(\mathrm{CH}_{3}\right)_{2}\right), 2.05$ (s, 3H, $\mathrm{CH}_{3}$ ); Anal. Calcd. for $\mathrm{C}_{19} \mathrm{H}_{20} \mathrm{~N}_{6} \mathrm{O}$ : C, 65.50; H, 5.79; N, 24.12. Found: C, 65.41; H, 5.68; N, $24.04 \%$.

1-Isonicotinoyl-3-methyl-4-phenyl-3a,4-dihydropyrazolo[3,4-c]pyrazole (4d). Yield 62\%, m.p. $264{ }^{0} \mathrm{C}$; IR (KBr) cm ${ }^{-1}: 3410$ (N-H str.), 3080 (C-H str., Ar-H), 2972, 2850 (C-H str., $\mathrm{CH}_{3}$ ), 1640 (C=O str.), 1590 (C=N str.); ${ }^{1} \mathrm{H}$ NMR $\left(\mathrm{CDCl}_{3}\right) \delta: 7.5-8.6(\mathrm{~m}, 9 \mathrm{H}, \mathrm{Ar}-\mathrm{H}), 6.2(\mathrm{~s}, 1 \mathrm{H}, \mathrm{NH})$, 4.28 (dd, 2H, CH-CH), $2.14\left(\mathrm{~s}, 3 \mathrm{H}, \mathrm{CH}_{3}\right)$; Anal. Calcd. for $\mathrm{C}_{17} \mathrm{H}_{15} \mathrm{~N}_{5} \mathrm{O}: \mathrm{C}, 66.87 ; \mathrm{H}, 4.95 ; \mathrm{N}$, 22.94. Found: C, 66.86; H, 4.84; N, 22.81\%.

Synthesis of 2-N-ethoxyphthalimido-3-(4-chloro phenyl)-6-isonicotinoyl-4-methyl-3,3adihydro pyrazolo[3,4-c]pyrazoles (6a). An equimolar mixture of (4a, $0.01 \mathrm{~mol})$ and phthalimidoxyethyl bromide $(\mathbf{5}, 0.01 \mathrm{~mol})$ in absolute ethanol was refluxed for $16 \mathrm{hrs}$. using pyridine $(0.02 \mathrm{~mol})$ as a base. Excess of solvent was removed in vacuo and the resultant product left was poured on crushed ice to obtain the product, which was filtered, dried and recrystallised from alcohol. 6a: Yield 64\%, m.p. $>300{ }^{0} \mathrm{C}$; IR (KBr) cm ${ }^{-1}: 3020$ (C-H str., Ar-H) , 2895 (C-H str., $\mathrm{CH}_{3}$ ), 1789, 1728, 1661 (C=O str.), 1601 (C=N str.), 1493 (N-O str.), 1079 (C-O str.), 788 (C-Cl str.); ${ }^{1} \mathrm{H}$ NMR $\left(\mathrm{CDCl}_{3}\right) \delta: 7.57-8.87$ (m, 12H, Ar-H), $4.48\left(\mathrm{t}, 2 \mathrm{H}, \mathrm{OCH}_{2}\right), 4.29(\mathrm{dd}, 2 \mathrm{H}$, $\mathrm{CH}-\mathrm{CH}), 3.64\left(\mathrm{t}, 2 \mathrm{H}, \mathrm{NCH}_{2}\right), 2.26\left(\mathrm{~s}, 3 \mathrm{H}, \mathrm{CH}_{3}\right) ; \mathrm{MS}: \mathrm{m} / \mathrm{z}: 528[\mathrm{M}]^{+}, 530[\mathrm{M}+2]^{+}, 459,344$, 325, 297, 185, 163, 150, 106, 80; Anal. Calcd. for $\mathrm{C}_{27} \mathrm{H}_{21} \mathrm{~N}_{6} \mathrm{O}_{4} \mathrm{Cl}$ : C, 61.31; H, 4.00; N, 15.89 . Found: C, 61.31; H, 3.86; N, 15.83\%. 
Compounds (6b-d) were also prepared in similar manner with change in reflux time. Their characteristic spectral and physical data are given below:

2- $\mathrm{N}$-Ethoxyphthalimido-6-isonicotinoyl-3-(4-methoxyphenyl)-4-methyl-3,3a-dihydro pyrazolo[3,4-c]pyrazoles (6b). Yield 61\%, m.p. $282{ }^{\circ} \mathrm{C}$; IR (KBr) cm ${ }^{-1}: 3080$ (C-H str., Ar-H) , 2890 (C-H str., $\mathrm{CH}_{3}$ ), 1780, 1720, 1650 (C=O str.), 1595 (C=N str.), 1481 (N-O str.), 1060 (C-O str.); ${ }^{1} \mathrm{H} \mathrm{NMR}\left(\mathrm{CDCl}_{3}\right) \delta: 7.50-8.89(\mathrm{~m}, 12 \mathrm{H}, \mathrm{Ar}-\mathrm{H}), 4.52\left(\mathrm{t}, 2 \mathrm{H}, \mathrm{OCH}_{2}\right), 4.28$ (dd, 2H, CH-CH), $3.74\left(\mathrm{~s}, 3 \mathrm{H}, \mathrm{OCH}_{3}\right), 3.54$ (t, $\left.2 \mathrm{H}, \mathrm{NCH}_{2}\right), 2.20\left(\mathrm{~s}, 3 \mathrm{H}, \mathrm{CH}_{3}\right) ; \mathrm{MS}: \mathrm{m} / \mathrm{z}: 524[\mathrm{M}]^{+}, 455,340,321$, 293, 185, 159, 150, 106, 80; Anal. Calcd. for $\mathrm{C}_{28} \mathrm{H}_{24} \mathrm{~N}_{6} \mathrm{O}_{5}$ : C, 64.11; H, 4.61; N, 16.02. Found: C, $64.03 ; \mathrm{H}, 4.44 ; \mathrm{N}, 15.84 \%$.

3-(4- $N, N$-Dimethylamino phenyl)- 2-N-ethoxyphthalimido-6-isonicotinoyl-4-methyl-3,3adihydro pyrazolo[3,4-c]pyrazoles (6c). Yield 59\%, m.p. $>300{ }^{0} \mathrm{C}$; IR (KBr) cm ${ }^{-1}: 3062(\mathrm{C}-\mathrm{H}$ str., Ar-H) , 2870 (C-H str., $\mathrm{CH}_{3}$ ), 1775, 1710, 1650 (C=O str.), 1592 (C=N str.), 1460 (N-O str.), 1070 (C-O str.); ${ }^{1} \mathrm{H}$ NMR $\left(\mathrm{CDCl}_{3}\right) \delta: 7.42-8.80$ (m, 12H, Ar-H), 4.50 (t, 2H, OCH $), 4.3$ (dd, $2 \mathrm{H}, \mathrm{CH}-\mathrm{CH}), 3.64\left(\mathrm{t}, 2 \mathrm{H}, \mathrm{NCH}_{2}\right), 2.82\left(\mathrm{~s}, 6 \mathrm{H}, \mathrm{N}\left(\mathrm{CH}_{3}\right)_{2}\right), 2.11\left(\mathrm{~s}, 3 \mathrm{H}, \mathrm{CH}_{3}\right) ; \mathrm{MS}: \mathrm{m} / \mathrm{z}: 537$ $[\mathrm{M}]^{+}, 468,353,334,306,185,172,150,106,80$; Anal. Calcd. for $\mathrm{C}_{29} \mathrm{H}_{27} \mathrm{~N}_{7} \mathrm{O}_{4}$ : C, 64.79; H, 5.06; N, 18.24. Found: C, 64.71; H, 4.96; N, 18.10\%.

2-N-Ethoxyphthalimido-6-isonicotinoyl-4-methyl-3-phenyl-3,3a-dihydropyrazolo-

[3,4clpyrazoles (6d). Yield 63\%, m.p. $>300{ }^{0} \mathrm{C}$; IR (KBr) cm ${ }^{-1}: 3081$ (C-H str., Ar-H), 1776, 1730, 1670 (C=O str.), 1585 (C=N str.), 1421 (N-O str.), 1050 (C-O str.); ${ }^{1} \mathrm{H}$ NMR $\left(\mathrm{CDCl}_{3}\right) \delta: 7.41$ $8.79(\mathrm{~m}, 13 \mathrm{H}, \mathrm{Ar}-\mathrm{H}), 4.66$ (t, 2H, OCH$), 4.25$ (dd, 2H, CH-CH), 3.59 (t, 2H, NCH$), 2.18$ (s, $\left.3 \mathrm{H}, \mathrm{CH}_{3}\right) ; \mathrm{MS}: \mathrm{m} / \mathrm{z}: 494[\mathrm{M}]^{+}$, 425, 310, 291, 263, 185, 129, 150, 106, 80; Anal. Calcd. for $\mathrm{C}_{27} \mathrm{H}_{22} \mathrm{~N}_{6} \mathrm{O}_{4}$ : C, 65.58; H, 4.48; N, 16.99. Found: C, 65.47; H, 4.33; N, 16.91\%.

\section{Acknowledgements}

The authors are thankful to the Head, Department of Chemistry, M. L. Sukhadia University, Udaipur for providing Laboratory facilities and to the Director, RSIC, CDRI, Lucknow, India for providing spectral and analytical data. Authors are grateful to Antimicrobial Research Laboratory, particularly Dr. Kanika Sharma, Department of Botany, M. L. Sukhadia University for evaluating anti-microbial activity and Prof. Erik De Clercq, Rega Institute for Medical Research, Katholicke University, Leuven, Belgium for carrying out antiviral and anticancer screening of the compounds. Two of the authors (Swati Ojha and Archita Bapna) are thankful to CSIR, New Delhi for providing necessary financial assistance. 


\section{References}

1. Govt. of India, Ministry of Health and Family Welfare; Indian Pharmacopoea Controller of Publication: Delhi, 1996, pp 408.

2. Mc Neill, L.; Allen, M.; Estrada, C.; Cook, P. Chest 2003, 123(1), 102.

3. Jasmer, R. M.; Sankonnen, J. J.; Blumberg, H. M.; Daley, C. L.; Bemardo, J.; Vittinaghoff, E.; King, M. D.; Dawamura, L. M.; Hopewell, P. C. Ann. Intern. Med. 2002, 137, 640.

4. World Health Organization: Geneva; WHO Global Tuberculosis Programme, 1997.

5. Alagarasamy, V.; Venkateshperumal, R.; Sathyabhama, S.; Vaishnavapriya, S.; Sakkarapandi, S.; Revathi, V.; Kalaiselvi, R.; Balamurugan, J.; Sevukarajan, M. Indian J. Heterocycl. Chem. 2002, 11, 327.

6. Lewis, R. The Rise of Antibiotic-Resistant Infections, FDA consumer magazine, Sept., 1995.

7. El-Kashef, H.; El-Emary, T.; Gasquet, M.; Timon-David, P.; Maldonado, J.; Vanello, P. Pharmazie 2000, 55, 572.

8. Taha, M.; Moukha-Chafiq, O.; Lazrok, H.; Vasseur, J.; Imbach, J. Nucleosides Nucleotides Nucleic Acids 2001, 20, 955.

9. Vicentini, C.; Forlani, G.; Manfrini, M.; Romagnoli, C.; Mares, D. J. Agric. Food Chem. 2002, 55, 4839.

10. Brozozonski, Z.; Saczawski, F. Eur. J. Med. Chem. 2002, 37, 709.

11. Hough, L.; Nalwalk, J.; Stadel, R.; Timmerman, H.; Leurs, R.; Paria, B.; Wang, X.; Dey, S. J. Pharmacol. Ex. Ther. 2002, 303, 14.

12. Jung, J. C.; Watkins, E. B.; Avery, M. A. Heterocycles 2005, 65, 77.

13. Palaska, E.; Aytemir, M.; Uzbay, T.; Erol, D. Eur. J. Med. Chem. 2001, 36, 539.

14. Bansal, E.; Srivastava, V. K.; Kumar, A. Eur. J. Med. Chem. 2001, 36, 81.

15. Ahn, J. H.; Kim, H. M.; Jung, S. H.; Kang, S. K.; Kim, K. R.; Rhee, S. D.; Yong, S. D.; Cheon, H. G.; Kim, S. S. Bioorg. Med. Chem. Lett. 2004, 14, 4461.

16. Tamilovi, Y. V.; Oknonnishnikova, G. P.; Shulishov, E. V.; Nefedov, O. M. Russ. Chem. Bt. 1995, 44, 2114.

17. Klimova, E. I.; Marcos, M.; Klimova, T. B.l Cecilio, A. T.; Ruben, A. T.; Lena, R. R. J. Organomet. Chem. 1999, 585, 106.

18. Bhaskarreddy, D.; Padmaja, A.; Ramanareddy, P. V.; Seenaiah, B. Sulfur Lett. 1993, 16, 227.

19. Bhaskarreddy, D.; Chandrasekhar, B. N.; Padmavathi, V.; Sumathi, R. D. Synthesis 1998, 491.

20. Groutas, W. C.; Brubaker, M. J.; Castrisos, J. C.; Crowley, J. P.; Schatz, E. J. J. Med. Chem. 1989, 32, 1607.

21. Alexander, M. S.; Stables, J. P.; Rutkowska, M. C.; Hurshthousu, M. B.; Hibbs, D. E.; Edafiogho, I. O.; Farrar, V. A.; Moore, J. A.; Scott, K. R. Eur. J. Med. Chem. 1996, 31, 787. 
22. Edafiogho, I. O.; Scott, K. R.; Moore, J. A.; Farrar, V. A.; Nicholson, J. M. J. Med. Chem. 1991, 34, 387.

23. Bhambi, D.; Salvi, V. K.; Jawahar, J. L.; Ojha, S.; Talesara, G. L. J. Sulfur Chem. 2007, 28, 155.

24. Ameta, U.; Ojha, S.; Bhambi, D.; Talesara G. L. ARKIVOC 2006, (xiii), 83.

25. Ahmed, M.; Dhakar, N.; Sharma, R.; Talesara, G. L. Indian J. Heterocycl. Chem. 2006, 16, 109.

26. Orndroff, W. R.; Pratt, D. S. Am. Chem. J. 1917, 47, 89.

27. Simmons, A. Practical Medical Microbiology, $14^{\text {th }}$ Edn., Churchill Livingston; Edinburgh, 1996; Vol. 11, p163.

28. Bisen, P. S.; Verma, K. Hand Book of Microbiology, $1^{\text {st }}$ Edn., CBS Publishers and Distributors: New Delhi, 1996.

29. De Clercq, E.; Descamps, J.; Verhelst, G.; Walker, R. T.; Jones, A. S.; Torrence, P. F.; Shugar, D. J. Infect. Dis. 1980, 141, 563.

30. De Clercq, E. Antimicrob. Agents Chemother. 1985, $28,84$. 